\title{
Immunopathological effects of aquaporin-4 igg in neuromyelitis optica spectrum disorders
}

\begin{abstract}
Neuromyelitis optica spectrum disorders (NMOSD) are central nervous system inflammatory disorders. Recently, autoimmunity against aquaporin-4 (AQP4) water channel is identified to be the underlying immunopathogenetic mechanism of the majority of NMOSD patients. This is evidenced by the detection of $\operatorname{IgG}$ autoantibodies against aquaporin-4 (AQP4-IgG) in the serum of $\sim 75 \%-80 \%$ of typical neuromyelitis optica patients. Detection of AQP4IgG is highly specific for NMOSD, facilitates diagnosis of NMOSD and their distinction from classical multiple sclerosis. Besides its diagnostic value, AQP4-IgG is likely directly pathogenic in NMOSD. This review focuses on the immunopathological effects of AQP4IgG in NMOSD.
\end{abstract}

Volume 5 Issue 4 - 2017

\author{
Koon Ho Chan, Roy Chun Laam NG, Leung \\ Wah Yick \\ Department of Medicine, The University of Hong Kong, Hong \\ Kong
}

Correspondence: Koon-Ho Chan, Department of Medicine, The University of Hong Kong, General Administration Office, Room 405B, 4/F, Professorial Block, Queen Mary Hospital, 102 Pok Fu Lam Road, Hong Kong, Tel +852-225-535-95, Fax 852297-4I I-7I,Email koonho@hku.hk

Received: January 01, 1970 | Published: May 29, 2017

\section{Introduction}

Neuromyelitis optica (NMO) and NMO spectrum disorders (NMOSD) are central nervous system inflammatory demyelinating disorders (CNS IDD) characterized by monophasic or relapsing optic neuritis (ON), acute myelitis (AM) and less commonly encephalitis. ${ }^{1}$ NMO-IgG, an autoantibody detected in serum of $73 \%$ of NMO but not in classical multiple sclerosis (CMS) patients. ${ }^{2}$ The autoantigen targeted by NMO-IgG is aquaporin-4 (AQP4), the major CNS water channel protein which is abundantly expressed in foot processes of astrocytes. ${ }^{3}$ The discovery of these IgG autoantibodies against aquaporin-4 (AQP4-IgG) detected in the serum of $~ 75 \%$ of NMO patients has clarified that NMOSD seropositive for AQP4-IgG are not CMS, but an autoimmune disorder affecting CNS astrocytes (astrocytopathy). NMOSD patients seropositive for AQP4-IgG have underlying autoimmunity against CNS astrocytic AQP4. A small proportion of patients with typical NMO (relapsing ON and extensive AM without brain involvement suggestive of CMS) and some with restricted forms such as relapsing extensive myelitis without $\mathrm{ON}$ are seronegative for AQP4-IgG. The immunopathogenesis of these patients are uncertain. A proportion of these patients $(\sim 20-25 \%)$ are seropositive for IgG autoantibodies against myelin oligodendrocyte glycoprotein (MOG-IgG). The pathogenesis of MOG-IgG positive patients await clarification from further studies, especially histopathological studies of affected CNS tissues.

In NMOSD, patients typically have monophasic or relapsing severe $\mathrm{AM}$ and $\mathrm{ON}$, with relatively less frequent brain involvement especially in the early phase, and CSF OCB are infrequent. ${ }^{1} \mathrm{NMO}$ patients typically have severe neurological disability after attacks of extensive myelitis, severe $\mathrm{ON}$ and brainstem encephalitis, ${ }^{4}$ no secondary progression and worse clinical outcome than CMS patients. ${ }^{1,5}$ Pathologically, spinal cord tissues of NMO patients exhibit necrosis in both gray and white matter, infiltrating leucocytes (macrophages, polymorphonuclear cells[neutrophils, eosinophils], and lymphocytes), activated microglia, demyelination, axonal loss, thickened hyalinized vessel walls with deposits of $\operatorname{IgM}$, IgG and complement activation products in a vasculocentric rim and rosette pattern. These hyalinized vessel walls with deposits of immunoglobulins and complement activation products in the characteristic vasculocentric rim and rosette pattern is not observed in lesions of CMS patients.

\section{Clinical usefulness of AQP4-IgG}

Distinction of NMOSD from CMS can be difficult especially in the early stage. ${ }^{5}$ In addition, brain involvement in NMO is increasingly recognized, and NMO patients with MRI brain lesions fulfilling criteria for CMS are reported. ${ }^{6}$ Early diagnosis of NMOSD is important as disability in NMOSD is relapse-related, ${ }^{1}$ hence relapse prevention is the key in treatment. Importantly, commonly used disease modifying drugs for relapsing MS including $\beta$-interferon, fingolimod and natalizumab may be ineffective or even harmful in NMOSD by precipitating development of extensive hemispheric lesions. ${ }^{7}$ Immunosuppression by azathioprine/mycophenolate mofetil and corticosteroids or B-cell depletion therapy (e.g. rituximab) should be initiated early in NMOSD to prevent relapses and disabilities. ${ }^{8}$ Detection of AQP4-IgG greatly facilitates early diagnosis and prompt treatment of NMOSD.

\section{Immunopathologic effects of AQP4-IgG}

AQP4-IgG is predominantly of IgG1 subclass, and binds to AQP4 abundantly expressed as transmembrane protein in astrocytic end feet processes at glial limitans abutting capillaries in pia, subpia and subependyma, and less abundantly in ependymal cells. ${ }^{3}$ AQP4 has six transmembrane regions and three extracellular loops with intracytoplasmic amino and carboxyl terminals. AQP4 exists as heterotetramers formed by the M1 and M23 isoforms (the 2 major isoforms). CNS AQP4 play important roles in functional integrity of blood-brain barrier (BBB) and CNS water homeostasis, regulating water transport across the interfaces between blood and brain, and between brain and CSF. ${ }^{9}$ Using sera from 32 NMOSD patients with high serum concentrations of AQP4-IgG, Iorio et al. ${ }^{10}$ showed that AQP4-IgG from NMOSD patients bind to extracellular loops, monomers, tetramers and high order orthogonal arrays of particles (OAP) of AQP4, and disease-specific epitopes reside in extracellular loop C more than in loop A or E. Importantly, AQP4-IgG bind most avidly to membrane AQP4 epitopes formed by loop interations within tetramers and intermolecular interations within OAP. ${ }^{10}$

AQP4-IgG are likely directly pathogenic in NMOSD. IgG from serum of AQP4-IgG positive NMO patients bound to extracellular region of membrane AQP4 of transfected HEK293 cells and triggered 
I. Internalization and endolysosomal degradation of bound AQP4, and

II. Complement activation with deposition of terminal lytic membrane attack complex, C9neo, causing cell lysis. ${ }^{11}$

QP4-IgG induced necrosis of cultured astrocytes in a complementdependent manner. AQP4-IgG binding to extracellular domains of AQP4 have isoform-specific outcomes, M1 isoform is completely internalized while M23 isoform aggregates into larger OAP via crosslinking and resist internalization. The Fc portion of the AQP4-IgG bound to these OAP triggers complement activation followed by astrocyte cytotoxicity, infiltration of lymphocytes and granulocytes (neutrophils, eosinophils) leading to intense neuro-inflammation, and tissue necrosis. ${ }^{12} \mathrm{An}$ in-vitro study that cultured rat astrocytes exposed to IgG from AQP4-IgG positive NMOSD patients developed into a reactive and inflammatory phenotype with increased expression of lipocalin-2 and a wide spectrum of cytokines, chemokines and stress response factors. ${ }^{13}$ The same group also reported that stimulation of astrocytes by AQP4-IgG led to NFKB-dependent inflammatory response which is one of the earliest events in NMOSD pathogenesis.

In-vivo studies revealed that passive transfer of $\mathrm{IgG}$ from AQP4-IgG positive NMOSD patients into laboratory animals with experimental autoimmune encephalomyelitis (EAE) induced by immunization with myelin proteins or adoptive transfer of myelin protein-reactive $\mathrm{T}$ lymphocytes enhanced CNS inflammation and worsened EAE score. Immunohistochemistry revealed loss of AQP4 and astrocytes in CNS lesions of the animals, mimicking pathologies of NMOSD patients. Intracerebral injection of IgG from AQP4IgG positive patients together with human complements into mouse brains induced NMO-like lesions with loss of AQP4 and astrocytes without preexisting inflammation. This study supports the important role of complement activation in neuro-inflammation and CNS tissue necrosis/damage in NMOSD. Rats immunized with complete Freud's adjuvant (CFA) only without myelin protein followed by passive transfer of $\operatorname{IgG}$ from AQP4-IgG positive NMOSD patients developed NMO-like lesions in spinal cord.

Colleagues and I studied the pathogenic role of AQP4-IgG in the absence of complement activation by passive transfer of IgG isolated from sera of NMO patients into mice (human $\operatorname{IgG}$ cannot activate mouse complements). Breakdown of $\mathrm{BBB}$ was induced before transfer of human IgG by subcutaneous CFA and intraperitoneal pertussis toxin (PTx). We observed that mice treated with IgG from AQP4-IgG positive NMOSD patients had areas of AQP4 loss in spinal cord while mice treated with IgG from AQP4-IgG negative NMOSD patients and IgG from healthy subjects did not. All mice had no clinical features of encephalomyelitis and no inflammatory cell infiltration, demyelination or loss of glial fibrillary acidic protein (GFAP) immunoreactivity. In addition, mice treated with IgG from AQP4-IgG positive NMOSD patients showed evidences of astrocytic activation with marked proliferation of foot processes. These suggest:

I. CFA and PTx alone lead to BBB breakdown,

II. AQP4-IgG from NMOSD patient's induced asymptomatic AQP4 loss and astrocytic activation without inflammatory cell infiltration, demyelination or astrocytic cytoxicity in the absence of complement activation, and

III. AQP4-IgG can activate astrocytes in the absence of complement activation. ${ }^{14}$

A recent animal study showed that chronic infusion of $\operatorname{IgG}$ from AQP4-IgG positive NMOSD patients to CSF of rats results in motor impairment of the animal associated with astrocyte alteration characterized by loss of AQP4, myelin basic protein, axons and reduced glutamate uptake but no immune cell infiltration, microglial activation or complement activation.

AQP4 expression is markedly reduced in cord lesions of NMOSD patients but preserved or increased in cord lesions of CMS patients. ${ }^{15}$ Recent histopathological studies further confirm that NMOSD have distinct pathologies from CMS. Although different pathologies are observed in different sites of lesions, AQP4 loss and astrocytic injury or cytotoxicity (loss) are the key pathologies in cord and brain lesions of NMOSD whereas normal AQP4 level and gliosis are observed in brain and cord lesions of CMS patients. ${ }^{15,16}$ Loss of AQP4 is always observed in CNS lesions of NMOSD patients, whereas loss of astrocyte, complement activation and necrosis are variable. Histopathological studies of brainstem lesions at the dorsal medulla (area postrema without intact BBB) from a small number of NMOSD patients revealed loss of AQP4, prominent astrocytic activation, lymphocytic infiltration, microglial reactivation, some complement activation products but no obvious axonal or neuronal injury. It is proposed that AQP4-IgG binding to astrocytic AQP4 at this site is followed by rapid internalization of antigen-antibody complex, possibly explained by predominantly AQP4 M1 expressed in this site. The astrocytes reacted with inflammatory response leading to inflammatory cell infiltration without obvious demyelination, axonal or neuronal injury. ${ }^{17}$

\section{Conclusion}

Current evidence suggests AQP4-IgG binding to astrocytic AQP4 are the initial step in neuroinflammation of NMOSD. AQP4-IgG in peripheral blood become accessible to CNS AQP4 at sites without intact BBB (such as the area postrema) or during inflammatory condition such as infection during which proinflammatory cytokines in circulation lead to breakdown of BBB. The astrocytic activation triggered upon binding of AQP4-IgG to AQP4 M1 and internalization of antigen-antibody complex release proinflammatory cytokines and chemokines leading to inflammatory cell infiltration. Other immune cells are activated with secretion of complements which will be activated especially at sites with high level of AQP4 M23 and hence OAP.

\section{Acknowledgments}

None.

\section{Conflicts of interest}

The authors declare no conflicts of interest.

\section{References}

1. Wingerchuk DM, Lennon VA, Lucchinetti CF, et al. The spectrum of neuromyelitis optica. Lancet Neurol. 2007;6(9):805-815.

2. Lennon VA, Wingerchuk DM, Kryzer TJ, et al. A serum autoantibody marker of neuromyelitis optica. Lancet. 2004;364(9451):2106-2112

3. Lennon VA, Kryzer TJ, Pittock SJ, et al. IgG marker of optic-spinal multiple sclerosis binds to aquaporin-4 water channel. J Exp Med. 2005;202(4):473-477.

4. Hinson SR, Lennon VA, Pittock SJ. Autoimmune AQP4 channelopathies and neuromyelitis optica spectrum disorders. Handbook of Clinical Neurology. 2016;133:377-403.

5. Pittock SJ, Lucchinetti CF. Neuromyelitis optica and the evolving spectrum of autoimmune aquaporin-4 channelopathies:a decade later. Ann N Y Aca Sci. 2016;1366(1):20-39. 
6. Pittock SJ, Lennon VA, Krecke K, et al. Brain abnormalities in patients with neuromyelitis optica (NMO). Arch Neurol. 2006;63(3):390-396.

7. Papeix C, Vidal JS, de Seze J, et al. Immunosuppressive therapy is more effective than interferon in neuromyelitis optica. Mult Scler. 2007;13(2):256-259.

8. Mandler RN, Davis LE, Jeffery DR, et al. Devic's neuromyelitis optica:a clinicopathological study of 8 patients. Ann Neurol. 1993;34(2):162168.

9. Ami-Moghaddam M, Ottersen OP. The molecular basis of water transport in the brain. Nat Rev Neurosci. 2003;4(12):991-1001.

10. Iorio R, Fryer JP, Hinson S, et al. Astrocytic autoantibody of neuromyelitis optica (NMO-IgG) binds to aquaporin-4 extracellular loops, monomers, tetramers and high order arrays. J Autoimmunity. 2013;40:21-27.

11. Hinson SR, Pittock SJ, Lucchinetti CF, et al. Pathogenic potential of IgG binding to water channel extracellular domain in neuromyelitis optica. Neurology. 2007;69(24):1-11.
12. Hinson SR, Romero MF, Popescu BFG, et al. Molecular outcomes of neuromyelitis optica (NMO)-IgG binding to aquaporin-4 in astrocytes. PNAS. 2012;109(4):1245-1250.

13. Howe CL, Kaptzan T, Magaña SM, et al. Neuromyelitis optica IgG stimulates an immunological response in rat astrocyte cultures. Glia. 2014;62(5):692-708.

14. Chan KH, Zhang R, Kwan JS, et al. Aquaporin-4 autoantibodies cause asymptomatic aquaporin -4 loss and activate astrocytes in mouse. $J$ Neuroimmunol. 2012;245:32-38.

15. Roemer SF, Lennon VA, Benarroch EE, et al. Pattern-specific loss of aquaporin-4 immunoreactivity distinguishes neuromyelitis optical from multiple sclerosis. Brain. 2007;130:1194-1205.

16. Lucchinetti CF, Guo Y, Popescu BF, et al. The pathology of an autoimmune astrocytopathy:lessons learned from neuromyelitis optica. Brain Pathology. 2014;24(1):83-97.

17. Popescu BF, Lennon VA, Parisi JE, et al. Neuromyelitis optica unique area postrema lesions: nausea, vomiting, and pathogenic implications. Neurology. 2011;76(14):1229-1237. 